\title{
ECG signals for human identification based on fiducial and non-fiducial approaches
}

\author{
Anwar E.Ibrahim ${ }^{1}$, Salah Abdel-Mageid ${ }^{2}$, Nadra Nada ${ }^{3}$ and Marwa A. Elshahed ${ }^{4}$ \\ Assistant Lecturer, Department of Physics, Faculty of Women for Arts, Sciences and Education, Ain Shams \\ University, Egypt ${ }^{1}$ \\ Professor, Department of Computer Engineering, Collage of Computer Science and Engineering, Taibah University, \\ Saudi Arabia $^{2}$ \\ Professor, Department of Physics, Faculty of Women for Arts, Sciences and Education, Ain Shams University, \\ Egypt $^{3}$ \\ Lecturer, Department of Physics, Faculty of Women for Arts, Sciences and Education, Ain Shams University, \\ Egypt $^{4}$
}

Received: 26-October-2019; Revised: 19-March-2020; Accepted: 21-March-2020

(C)2020 Anwar E.Ibrahim et al. This is an open access article distributed under the Creative Commons Attribution (CC BY) License, which permits unrestricted use, distribution, and reproduction in any medium, provided the original work is properly cited.

\begin{abstract}
Biometric systems are mostly used for human identification and authentication. Recent developments have shown that ECG human identification can be used as a powerful tool as it gives more reliable and accurate results. In this paper, a proposed human identification system based on ECG as a biometric is presented with different feature extraction methods. Different feature extraction methods such as Daubechies wavelet ('db3', 'db8' and 'db10'), Symlets wavelet 'sym7' and Biorthogonal wavelet 'bior2.6' are exploited in this work. A combination of radial basis function (RBF) neural network and Backpropagation (BP) neural network is used as a classifier. The proposed system gives an identification rate of $98.41 \%$ with Daubechies wavelet 'db8'. In addition, the identification rate for Daubechies wavelet ('db3'and 'db10'), Symlets wavelet 'sym7' and Biorthogonal wavelet 'bior2.6' increases when $R-R$ intervals are added as fiducial features of the non-fiducial features.
\end{abstract}

\section{Keywords}

Biometric, ECG signal, Fiducial, Non-fiducial.

\section{Introduction}

Humans recognize each other according to their various characteristics. For example, a father can recognize his daughter by her face when he meets her and by her voice when she speaks to him. In information technology, biometrics is defined as a method to measure and analyze human body characteristics such as iris, DNA, fingerprints, facial patterns, retina and hand measurements, and so on. For authentication purposes, biometrics is a method of recognizing humans depend on unique physical or behavioral characteristics $[1,2]$.

Not all human traits can be used as a biometric, but it should be characterized by:

*Author for correspondence
- Universality: all persons must possess the biometric trait.

- Distinctiveness: the biometric traits should be unique for each person as DNA.

- Permanence: a good biometric trait is invariant or changes slowly over time.

- Collectability: a biometric trait should be easily collected quantitatively.

In addition, the biometric system should be characterized by:

- Acceptability: The extent for users to accept the daily using of biometric identifiers.

- Performance: according to requirements, accuracy, speed, and robustness.

- Circumvention: it should counteract to fraudsters [3]. 
Biometric system is a pattern recognition system which captures biometric characteristic from the person then extracts features from the biometric and compares the features with feature set(s) stored in the database at the end gives an action depend on the result of the comparison. Iris recognition, fingerprint and face recognition are considered external physiological biometrics, the problem of these biometrics is that it is easy to mimic and fake finger, face photos and iris. Therefore, a new internal physiological biometric such as ECG is required to solve this problem $[4,5]$.

Electrocardiogram (ECG or sometimes called aan EKG ((Electrokardiogram)) is a method to measure the electrical activity of the heart over time, which is recorded by electrode sensors placed on the body surface [6]. Biel et al. (1999) first provided experimental evidence that ECG contained information specific to persons. The different structure of the heart between subjects affects the shape of the ECG signal; therefore, this permits for a biometric system to use the electrocardiogram signal for human identification purposes $[7,8]$. Each beat in the ECG signal contains three main waves, the $\mathrm{P}$ wave, the QRS complex and the $\mathrm{T}$ wave [9]. ECG based biometric systems can be classified into two categories. Fiducial (analytical) features based systems that use fiducial points such as the three peak points $(\mathrm{P}, \mathrm{R}$, and $\mathrm{T})$, two valleys ( $\mathrm{Q}$ and $\mathrm{S}$ ) and the six onsets \& offsets for the three waves to extract features. Non-fiducial (appearance) features based systems usually investigate the ECG frequency content. Non-fiducial features are like wavelet coefficients, discrete cosine transform coefficient, and so on. No fiducial detection is needed except for the $\mathrm{R}$ peak and for some non-fiducial approaches; no fiducial detection is required [10].

There are several existing methods for using the ECG signal as a biometric. These methods used various techniques that are linear predictive coding (LPC), wavelet packet decomposition (WPD), discrete wavelet transform (DWT), and autocorrelation and discrete cosine transform (AC/DCT) as feature extraction, neural network, hidden Markov model (HMM) with the (HTK) recognition toolkit using a Bakis model with one Gaussian, MLP neural network, linear discriminant analysis (LDA) and support vector machine as classifiers [8, 11-18].

In this paper, a proposed algorithm based on nonfiducial and fiducial features is presented with different feature extraction methods to increase the robustness and accuracy of ECG biometric systems. In our previous work, we make a comparative study between RBF and BP. From the results, the RBF gives higher accuracy than $\mathrm{BP}$ neural network and the combination of the two classifiers RBF and BP improved the obtained identification rate, so the combination of the two classifiers is used in this work.

This paper is organized as follows. Some related work is described in section 2. The proposed system architecture is described in section 3. In section 4, experiments and results are shown. Finally, section 5, conclusions and future work.

\section{Related work}

Some non-fiducial and fiducial studies are presented below. The authors in [8], proposed a new ECG biometric system based on Linear Predictive Coding (LPC) and Wavelet Packet Decomposition (WPD) as feature extraction and neural network used as a classifier. The results show that the recognition rate for using LPC was $100 \%$ and $92 \%$ for using WPD. ECG signals of 15 healthy students were used in this study. Moreover, there is no sufficient dataset for learning and testing also all the used subjects are males and have a small range of age.

In [11], an ECG biometric system based on fiducial features was proposed. Ten morphological features and sixty Hermite polynomial expansion (HPE) coefficients are extracted from each heartbeat. The classification was applied using hidden Markov model (HMM) with the (HTK) recognition toolkit using a Bakis model with one Gaussian. 18 healthy persons form the MIT_BIH database was used. The recognition rate is $96.7 \%$ for the (HPE) coefficients and $99 \%$ for the combination between ten morphological features and sixty HPE coefficients. This work achieved high accuracy; nevertheless, the used dataset is not sufficient for training and testing also the system used only the healthy subjects.

The authors in [12], presented different feature extraction methods for the classification of an electrocardiogram. Fiducial features extracted related to the QRS complex using Tompkin's algorithm also discrete wavelet transform (db5) used as feature extraction. The multilayer perceptron neural network was used for classification. Eight normal subjects were used in this study. The results show that the average classification accuracy of using fiducial features was $71.80093 \%, 62.69305 \%$ for using nonfiducial features and $98.0556 \%$ for using hybrid 
features. This work achieved high accuracy; however, the used dataset is not sufficient for training and testing. The test for unauthorized persons is unclear. The authors in [13], proposed an ECG biometric system. Template matching technique used in the authentication process and linear support vector machine algorithm used for the classification. Forty-four subjects were downloaded from physionet databases, 22 of the subjects were authorized while the other 22 are not authorized users. The accuracy rate was $97.2 \%$ in the authentication process, $98.6 \%$ of the classification task. However, there is no enough dataset for learning and testing.

In [14], an ECG biometric identification system was proposed. Statistical features like mean, variance and standard deviation, other features like PQRST wave's amplitude and DWT mean intervals are extracted from the recorded ECG. Artificial neural network (ANN) was used for classification. Ten healthy subjects were used in this study. The accuracy of the identification system is $100 \%$. This work achieved high accuracy; however, the used dataset is not sufficient for training and testing, also the system used only the healthy subjects. There is no test for unauthorized persons.

The authors in [15], proposed a biometric identification method based on three different feature sets. The first feature set was extracted using the AC/DCT method, while the second feature set (Cepstral features) was extracted using, adapting Mel-Frequency Cepstral Coefficients (MFCC) extraction algorithm, and for the third feature set the authors determined the QRS beats for each ECG frame. Both LDA and 5-NN were used as classifiers. Three databases were used, the first database contains 30 healthy subjects, while the second database and third databases were composed of 45 and 60 subjects (these databases were created by increasing the number of subjects in the first database from 30 to 45 and then from 45 to 60 . The recognition rate was $100 \%$ (30 out of 30), $100 \%$ (45 out of 45) and $98.33 \%$ (59 out of 60 ) for 30,45 and 60 subjects respectively. This work achieved high accuracy; however, the accuracy was calculated for the known subjects only and there is no test for unauthorized subjects. Also, the system used only healthy subjects. In [16], an ECG biometric identification method based on a two-dimensional convolution neural network was proposed. AC/DCT features and Cepstral features were extracted from overlapping ECG signals. This work used 42 subjects that were selected from the PTB diagnostic ECG database. The proposed system achieved accuracy $88.57 \%$ and an identification rate $90.48 \%$ (38 out of 42 ), however, the accuracy was calculated for the known subjects only and there is no test for unauthorized subjects.

\section{The system architecture of proposed scheme}

The proposed identification system contains three main steps pre-processing, feature extraction and reduction, and classification, as shown in Figure 1. The pre-processing steps are explained as follows; the dataset is filtered so we used it directly. Then, The Pan and Tompkins algorithm was applied to $\mathrm{R}$ peak detection. We select $10 \mathrm{R}-\mathrm{R}$ cycles for each person is used in training. Since feature vectors must have an equal length each cycle length was fixed at 200 samples. The amplitude of all points for each R$\mathrm{R}$ cycle was normalized in the range of [0-1].

Discrete Wavelet Decomposition was applied as a feature extraction method to select R-R cycles. In this paper, we present a comparative study between some mother wavelets, Daubechies wavelet ('db3', 'db8' and 'db10'), Symlets wavelet 'sym7'and Biorthogonal wavelet 'bior2.6'. In this work, the number of decomposition levels is chosen to be 5 . The resulted wavelet coefficient structure contains six parts (d1 to $\mathrm{d} 5$, a5), five parts (d1 to d5) for the coefficients resulted from the details region for each level and one part (a5) for the coefficients resulted from the remaining approximation region in the last level.

Figure 2 shows the 5-level discrete wavelet decomposition using Daubechies wavelet 'db3'. The number of the wavelet coefficients after 5-level discrete wavelet decomposition is 223 coefficients. After $(\mathrm{d} 1, \mathrm{~d} 2)$ are discarded we use 68 coefficients. To improve the human identification rate, we used a combination of RBF and BP neural network as a classifier. ECG-ID database is used in our experiment. This database contains 310 ECG recordings, obtained from 90 persons. It consists of 44 men and 46 women aged from 13 to 75 years. Each recording contains ECG lead I, recorded for 20 seconds, digitized at $500 \mathrm{~Hz}$. The number of records for each subject varies from 2 to 20 collected periodically over 6 months [17]. In our study 65 subjects are used to train the ANN. Forty subjects are used for testing. Among these 32 subjects are used for testing known (authorized) persons, and 8 subjects are used for testing unknown (unauthorized) persons. In our experiments, we used a record for training and another one for testing. 
Anwar E.Ibrahim et al.

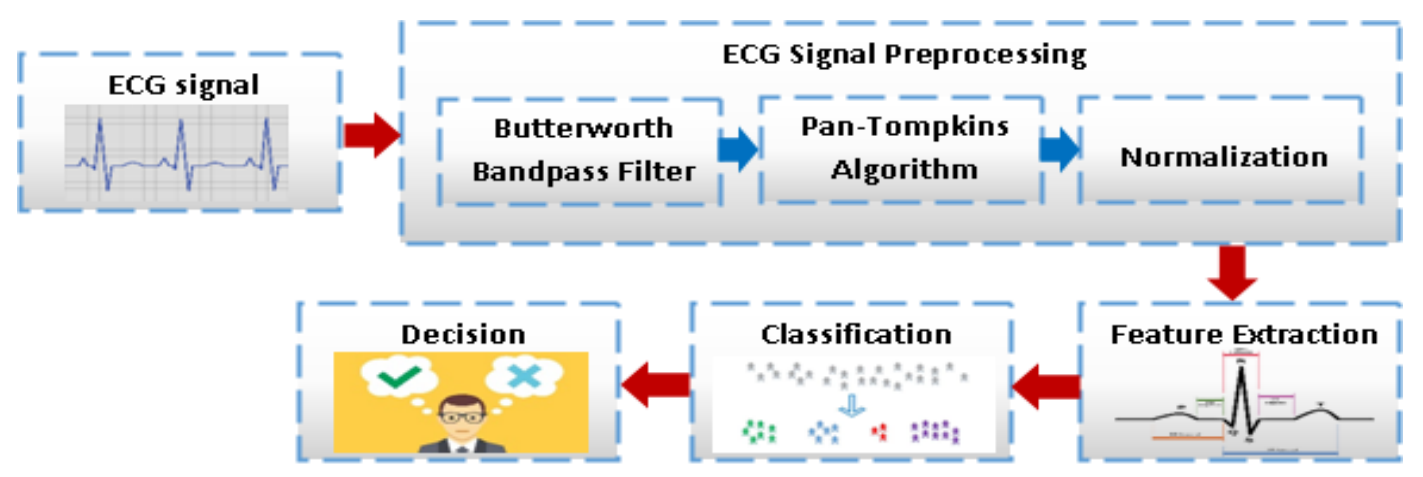

Figure 1 Block diagram of proposed system

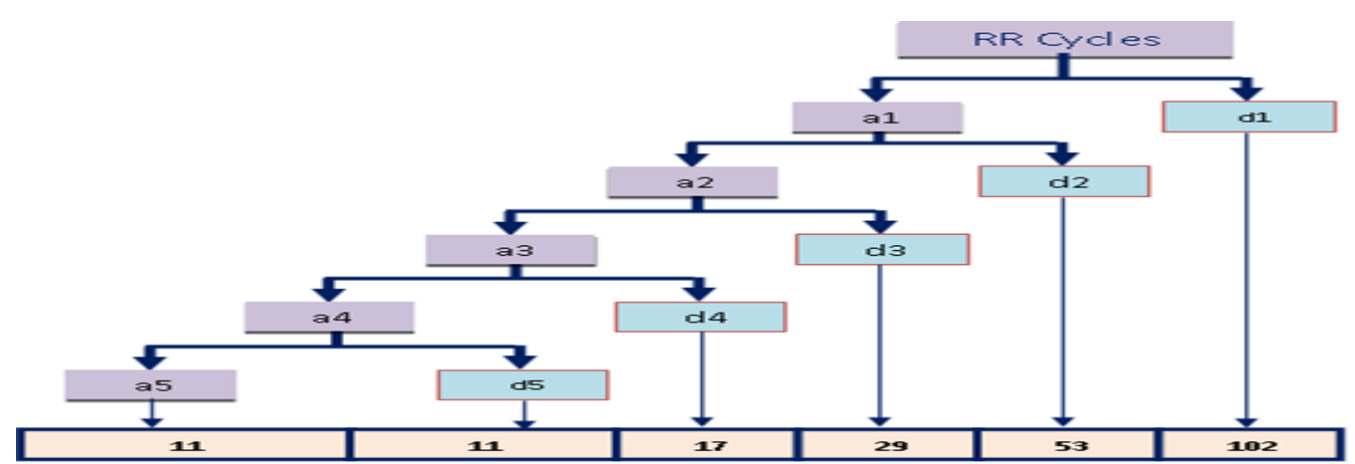

Figure 2 The 5-level discrete wavelet decomposition using Daubechies wavelets 'db3'

\section{Experiments and results}

The proposed system performance was determined by the accuracy, False Acceptance Rate (FAR) and False Rejection Rate (FRR), also some metrics such as recall, precision and f-score were calculated. At first Daubechies wavelet ('db3') was used as a feature extraction method which achieves identification rate $91.52 \%$. The experiment was repeated using the other feature extraction methods such as Daubechies wavelet ('db8') which gives $98.41 \%$, while ( 'db10') gives $93.53 \%$, also Symlets wavelet 'sym7' gives 95.07\% , and Biorthogonal wavelet 'bior2.6' achieves $91.8 \%$. Table 1 contains the system performance for the used wavelets.

Daubechies wavelet family is similar in shape to QRS complex and its energy spectrum is concentrated around low frequencies. The result gives that Daubechies wavelet 'db8' achieves the higher identification rate $(98.41 \%)$ than the others used methods. To improve the accuracy of Daubechies wavelet ('db3', 'db8' and 'db10'), Symlets wavelet 'sym7' and Biorthogonal wavelet 'bior2.6' we proposed a hybrid identification system that used the non-fiducial and fiducial features. We calculate the
$\mathrm{R}-\mathrm{R}$ interval as a fiducial feature, in addition to the wavelet coefficients of each mother wavelet. R-R interval is the time interval between two successive $\mathrm{R}$ - peaks.

Table 2 contains the accuracy, (FAR), (FRR), recall, precision and f-score for Daubechies wavelet ('db3', 'db8' and 'db10'), Symlets wavelet 'sym7' and Biorthogonal wavelet 'bior2.6' after we add the R-R intervals to the wavelet coefficients.

Figure 3 shows a comparison between the f-score value for 'db3', 'db8', 'db10', 'sym7' and 'bior2.6' before and after we add the R-R intervals to the wavelet coefficients. From the results, we found that the identification rate for Daubechies wavelet ('db3'and 'db10'), Symlets wavelet 'sym7' and Biorthogonal wavelet 'bior2.6' is increased after the $\mathrm{R}-\mathrm{R}$ intervals are added as a fiducial feature to the non-fiducial features. The following Table 3 presents a comparison between our proposed work and some previous related works using the same dataset (ECGID). 
Table 1 Comparison between the used feature extraction methods

\begin{tabular}{lllllll}
\hline $\begin{array}{l}\text { Feature extraction } \\
\text { methods }\end{array}$ & $\begin{array}{l}\text { Subject recognition } \\
\text { accuracy }(\boldsymbol{\%})\end{array}$ & $\begin{array}{l}\text { FRR } \\
(\boldsymbol{\%})\end{array}$ & $\begin{array}{l}\text { FAR } \\
(\boldsymbol{\%})\end{array}$ & $\begin{array}{l}\text { Precision } \\
(\boldsymbol{\%})\end{array}$ & $\begin{array}{l}\text { Recall } \\
(\boldsymbol{\%})\end{array}$ & $\begin{array}{l}\text { F-score } \\
(\boldsymbol{\%})\end{array}$ \\
\hline $\mathrm{db} 3$ & 87.5 & 12.5 & 0 & 100 & 84.37 & 91.52 \\
\hline $\mathrm{db} 8$ & 97.5 & 2.5 & 0 & 100 & 96.87 & 98.41 \\
\hline $\mathrm{db} 10$ & 90 & 7.5 & 2.5 & 96.67 & 90.6 & 93.53 \\
\hline $\mathrm{sym} 7$ & 92.5 & 7.5 & 0 & 100 & 90.6 & 95.07 \\
\hline bior2.6 & 87.5 & 10 & 2.5 & 96.55 & 87.5 & 91.8 \\
\hline
\end{tabular}

Table 2 The accuracy, FAR, FRR, recall, precision and f-score for 'db3','db8', 'db10', 'sym7' and 'bior2.6' after we add the R-R intervals to the wavelet coefficients

\begin{tabular}{lllllll}
\hline $\begin{array}{l}\text { Feature extraction } \\
\text { methods }\end{array}$ & $\begin{array}{l}\text { Subject recognition } \\
\text { accuracy } \mathbf{( \% )}\end{array}$ & $\begin{array}{l}\text { FRR } \\
(\mathbf{\%})\end{array}$ & $\begin{array}{l}\text { FAR } \\
(\boldsymbol{\%})\end{array}$ & $\begin{array}{l}\text { Precision } \\
(\boldsymbol{\%})\end{array}$ & $\begin{array}{l}\text { Recall } \\
(\boldsymbol{\%})\end{array}$ & $\begin{array}{l}\text { F-score } \\
(\boldsymbol{\%})\end{array}$ \\
\hline $\mathrm{db} 3$ & 90 & 10 & 0 & 100 & 87.5 & 93.33 \\
\hline $\mathrm{db} 8$ & 97.5 & 2.5 & 0 & 100 & 96.87 & 98.41 \\
\hline $\mathrm{db} 10$ & 92.5 & 5 & 2.5 & 96.77 & 93.75 & 95.23 \\
\hline $\mathrm{sym} 7$ & 97.5 & 2.5 & 0 & 100 & 96.87 & 98.41 \\
\hline bior2.6 & 92.5 & 7.5 & 0 & 100 & 90.6 & 95.07 \\
\hline
\end{tabular}

Table 3 Comparison between our proposed work and some previous works

\begin{tabular}{lllll}
\hline Author & Feature extraction method & Classification & & Accuracy \\
\hline Dar, et.al (2015) [18] & $\begin{array}{l}\text { Discrete Wavelet Transform (DWT) } \\
\text { and Heart Rate Variability (HRV) } \\
\text { based features }\end{array}$ & Random Forests & $83.88 \%$ \\
\hline Dar, et.al (2015) [19] & Discrete Wavelet Transform (DWT) & $\begin{array}{l}\text { single nearest neighbor } \\
\text { classifier (KNN) }\end{array}$ & $82.3 \%$ \\
\hline $\begin{array}{l}\text { Salloum and Kuo (2017) } \\
{[20]}\end{array}$ & Discrete Wavelet Transform (DWT) & $\begin{array}{l}\text { recurrent neural networks } \\
\text { (RNNs) }\end{array}$ & $\begin{array}{l}\text { Radial basis function (RBF) + } \\
\text { back propagation (BP) neural } \\
\text { network }\end{array}$ \\
\hline Proposed work & & & \\
\hline
\end{tabular}

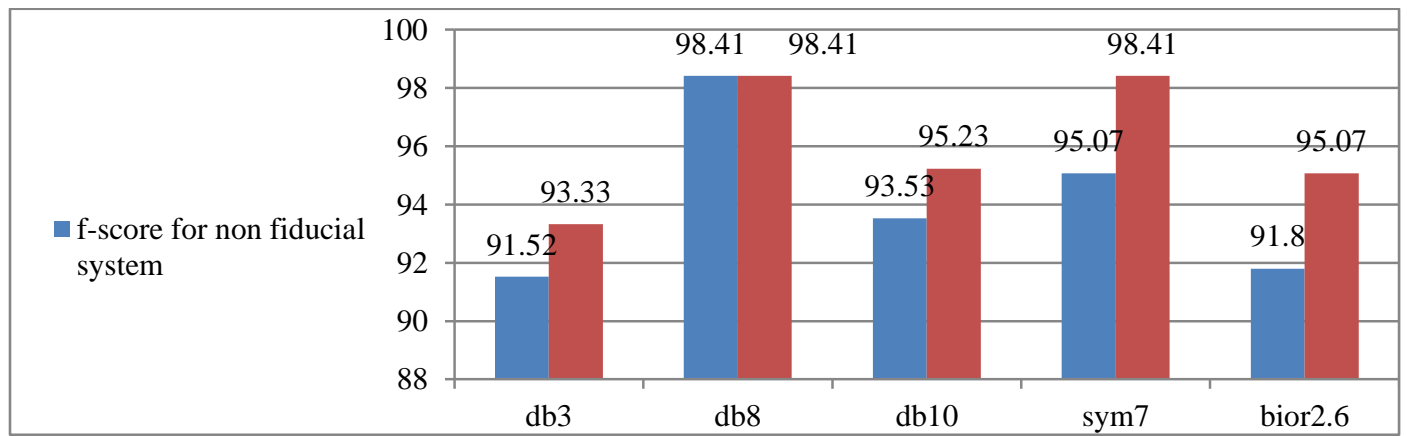

Figure 3 The f-score value for ' $\mathrm{db} 3$ ','db8', 'db10', 'sym7' and 'bior2.6' before and after we add the R-R intervals to the wavelet coefficients

\section{Conclusion}

This paper proposed a non-fiducial identification system and a hybrid identification system which uses the fiducial and non-fiducial features based on ECG signal as biometric. A comparative study is presented for different feature extraction methods. The result gives that Daubechies wavelet 'db8' achieves the higher identification rate $(98.41 \%)$ than the others used methods. In addition, we found that the identification rate for Daubechies wavelet ('db3'and 'db10'), Symlets wavelet 'sym7' and Biorthogonal wavelet 'bior2.6' is increased after adding the R-R intervals as fiducial features to the non-fiducial features.

\section{Acknowledgment}

None. 
Conflicts of interest

The authors have no conflicts of interest to declare.

\section{References}

[1] http://citeseerx.ist.psu.edu/viewdoc/download?doi=10. 1.1.24.3840\&rep $=$ rep $1 \&$ type $=$ pdf. $\quad$ Accessed 20 August 2019.

[2] Choudhary J. Survey of different biometrics techniques. International Journal of Modern Engineering Research. 2012; 2(5):3150-5.

[3] Hassan Z, Gilani SO, Jamil M. Review of fiducial and non-fiducial techniques of feature extraction in ECG based biometric systems. Indian Journal of Science and Technology. 2016; 9(21):23-6.

[4] Sareen P. Biometrics-introduction, characteristics, basic technique, its types and various performance measures. International Journal of Emerging Research in Management and Technology. 2014; 3:109-9.

[5] Verma N, Rana M. Biometric identification of person using ECG signal. Journal of Chemical, Biological and Physical Sciences. 2013; 4(1).

[6] Wahabi S. Variability in ECG biometrics: state of the art and subspace methods (Doctoral dissertation, University of Toronto).2015.

[7] Belgacem N, Nait-Ali A, Fournier R, Bereksi-Reguig F. ECG based human authentication using wavelets and random forests. International Journal on Cryptography and Information Security. 2012; 2(2):111.

[8] Loong JL, Subari KS, Besar R, Abdullah MK. A new approach to ECG biometric systems: a comparative study between LPC and WPD systems. World Academy of Science, Engineering and Technology. 2010; 68(20):759-64.

[9] Nawal M, Purohit GN. ECG based human authentication: a review. International Journal of Emerging Engineering Research and Technology. 2014; 2(3):178-85

[10] Tantawi M, Revett KE, Salem AB, Tolba MF. ECG based biometric recognition using wavelets and $\mathrm{RBF}$ neural network. Recent advances in information science 2013 (pp. 100-4).

[11] Rabhi E, Lachiri Z. Biometric personal identification system using the ECG signal. In computing in cardiology 2013 (pp. 507-10). IEEE.

[12] Gawande PS and Ladhake SA. An impact of different feature extraction methods on classification of electrocardiogram. International Journal of Advanced Research in Computer Science and Software Engineering. 2015; 5(7):667-70.

[13] Shdefat AY, Joo MI, Choi SH, Hee-Cheol K. Utilizing ECG waveform features as new biometric authentication method. International Journal of Electrical and Computer Engineering. 2018; 8(2):65865.

[14] Yadav N, Duhan M and Rose A. Biometric human recognition using ECG signals. International Advanced Research Journal in Science, Engineering and Technology. 2017; 4(6):168-71.
[15] Guven G, Gürkan H, Guz U. Biometric identification using fingertip electrocardiogram signals. Signal, Image and Video Processing. 2018; 12:933-40.

[16] Hanilçi A, Gürkan H. ECG biometric identification method based on parallel 2-D convolutional neural networks. Journal of Innovative Science and Engineering. 2019; 3(1):11-22.

[17] https://archive.physionet.org/cgi-bin/atm/ATM. Accessed 20 August 2019.

[18] Dar MN, Akram MU, Shaukat A, Khan MA. ECG based biometric identification for population with normal and cardiac anomalies using hybrid HRV and DWT features. In international conference on IT convergence and security 2015 (pp. 1-5). IEEE.

[19] Dar MN, Akram MU, Usman A, Khan SA. ECG biometric identification for general population using multiresolution analysis of DWT based features. In second international conference on information security and cyber forensics (InfoSec) 2015 (pp. 5-10). IEEE.

[20] Salloum R, Kuo CC. ECG-based biometrics using recurrent neural networks. In international conference on acoustics, speech and signal processing 2017 (pp. 2062-6). IEEE.

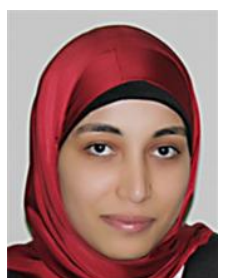

Anwar E.Ibrahim received her B.Sc. in Physics and Computer Science and M.Sc. in Computer Science from Faculty of Women for Arts, Sciences and Education, Ain Shams University, Egypt in 2011and 2016, respectively. She is currently a Ph.D. student and Assistant Lecturer in Physics Department, Faculty of Women for Arts, Sciences and Education, Ain Shams University, Egypt. Her current research interests include Artificial Intelligence, Biometrics, and Machine Learning.

Email: anwar.elbayomi@women.asu.edu.eg

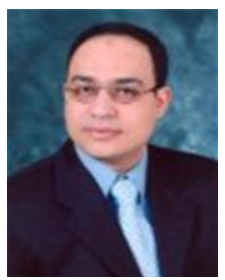

Salah M. Abdel Mageid received his M.S. and Ph.D. in Systems and Computers Engineering from Al-Azhar University in 2002 and 2005, respectively. He is currently a Professor in Department of Computer Engineering, Collage of Computer Science and Engineering, Taibah University, Saudi Arabia. He performed his post-doctoral research in 2007 and 2008 in the Computer Science and Engineering Department, School of Engineering, the Southern Methodist University in Dallas, TX, USA. He was a member of TEMPO (Tool for Extensive Management and Performance Optimization) project in Cairo University and Vodafone Egypt as an industrial partner in 2014 and 2015. His research interests include Mobile Computing, Cellular Networks, Sensor Networks, Cognitive Radio Networks, Vehicular Ad-hoc Networks, Big Data and Data Analysis, Internet Services and Applications. Email: sabdelmageid@taibahu.edu.sa 


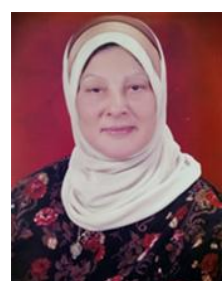

Nadra Nada received her B.Sc. in Sciences of the General Mathematics from Faculty of Women for Arts, Sciences and Education, Ain Shams University, Egypt and Ph.D. in Spectral Measurements from Martin-Lutheruniversity, Germany, in 1964 and 1975, respectively. She is currently a

Professor of atomic and molecular spectra physics in Physics Department, Faculty of Women of Arts, Sciences and Education, Ain Shams University, Egypt.

Email: nadra.nada@womenasu.edu.eg

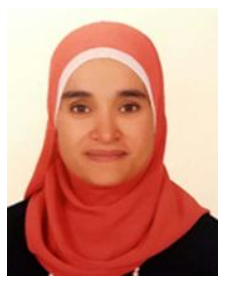

Marwa A. Elshahed received her B.Sc. in physics and computer science, M.Sc. and Ph.D. in computer science from Faculty of Women for Arts, Sciences and Education, Ain Shams University, Egypt in 2001, 2006 and 2010, respectively. She is currently lecturer in Computer Science, Faculty of Women for Arts, Sciences and Education, Ain Shams University, Egypt. Her current research interests include Artificial Intelligence, Biometrics, and Machine Learning. Email:marwa.ali@womenasu.edu.eg 